\title{
Definir asma para estudos epidemiológicos: essa meta pode ser alcançada?
}

\author{
MÁRCIA MARGARET MENEZES PIZZICHINI
}

Diferenças e similaridades na prevalência de asma entre paises e dentro de um mesmo país podem fornecer informações relevantes sobre o comportamento dessa condição e sobre fatores de risco suscetiveis de prevenção. ${ }^{(1)}$ Contudo, essas diferenças podem simplesmente resultar do emprego de definições distintas de asma ou de diferentes instrumentos (por exemplo: questionários) de medida. Adicione-se a isto o fato de ainda não existir uma definição universalmente aceita e que englobe os diversos fenótipos da asma de crianças e de adultos.

Definir asma é sempre objeto de discussão. Mais recentemente, os consensos têm definido asma de forma cuidadosa e elaborada, mas o resultado é ainda insatisfatório. ${ }^{(2-3)} \mathrm{A}$ falta de um marcador biológico ou fisiológico exclusivo da asma, ou ainda a falta de especificidade dos sintomas de asma e a variabilidade de expressão clínica da doença entre os pacientes ou em um mesmo paciente, tornam essas definições insatisfatórias por serem mais descritivas do que assertivas. Essas dificuldades na definição de asma explicam em grande parte as dificuldades em se investigar a epidemiologia da doença.

Até o presente, o estudo epidemiológico da asma tem se fundamentado principalmente no emprego de questionários. Nesse sentido, o questionário do International Study of Asthma and Allergies in Childhood (ISAAC) - Fase 1, concebido para estabelecer a prevalência mundial de asma e de sintomas respiratórios em crianças com idades entre seis e sete anos e entre treze e catorze anos, foi o primeiro a trazer uniformidade na coleta dos dados nessas faixas etárias, a despeito das diferenças culturais e das barreiras de linguagem. ${ }^{(4)} 0$ módulo de asma do questionário ISAAC Fase 1 contém quatro questões referentes à ocorrência de sintomas de asma (sibilos alguma vez, sibilos no último ano, sibilos desencadeados pelo exercício e tosse seca noturna), três sobre a gravidade dos sintomas (número de episódios ou crises de sibilos por ano, sibilância noturna e sibilância dificultando a fala) e uma questão sobre diagnóstico de asma. Os resultados do ISAAC Fase 1 demonstraram ampla variação na prevalên- cia de asma e de sintomas de asma entre os diferentes paises e entre regiões de um mesmo país. ${ }^{(5-6)}$ No Brasil, os dados do ISAAC Fase 1 foram coletados nas cidades de São Paulo (SP), ${ }^{(7)}$ Curitiba (PR), ${ }^{(8)}$ Porto Alegre (RS), ${ }^{(6)}$ Itabira (MG), ${ }^{(9)}$ Recife (PE), ${ }^{(10)}$ Campos Gerais (MG) ${ }^{(11)}$ e Florianópolis (SC), ${ }^{(12)}$ entre outras. 0 diagnóstico de asma alguma vez nestas cidades variou entre $4,8 \%$ e $26,4 \%$. A magnitude e a variabilidade destas prevalências espelham o que tem ocorrido no panorama mundial. ${ }^{(6-7)}$

0 estudo epidemiológico da asma em adultos oferece dificuldades adicioniais as quais incluem a duração da doença, tipo e tempo de tratamento, exposição ocupacional, exposição ambiental, tabagismo, co-morbidades, etc. 0 European Community Respiratory Health Survey (ECRHS) é um questionário concebido com o objetivo de uniformizar a investigação epidemiológica de sintomas respiratórios sugestivos de asma, presença de alergias e uso de tratamento em adultos com idades entre 20 e 44 anos. ${ }^{(13)}$ Similarmente ao estudo ISAAC Fase 1, os resultados do ECRHS demonstraram ampla variação na prevalência de sintomas de asma entre os países estudados. Este ou outros questionários padronizados para avaliar a prevalência de asma em adultos ainda não foram utilizados de modo sistemático no Brasil até o momento.

Neste número do Jornal Brasileiro de Pneumologia, Maçãira et al. ${ }^{(14)}$ apresentam os resultados de um estudo cujo objetivo foi validar um método de construção de um escore para o módulo de asma do questionário padronizado escrito do ISAAC e propor um ponto de corte capaz de discriminar adultos asmáticos utilizando como padrão ouro o diagnóstico clínico e funcional. Para tanto, os autores selecionaram aleatoriamente 40 asmáticos adultos (asma leve 15\%, asma moderada $45 \%$, asma grave $25 \%$ e não classificada 15\%) e 38 controles. Asmáticos e controles foram recrutados dos ambulatórios de Pneumologia e Clínica Médica, respectivamente, do Hospital das Clínicas da Faculdade de Medicina da Universidade de São Paulo. 0 escore de sintomas foi construído através de notas atri- 
buídas a cada uma das questões do módulo de asma do questionário ISAAC por vinte especialistas, distribuidos entre pneumologistas, alergistas e clínicos gerais (todos com experiência em asma). As notas variaram de zero a 2 de acordo com a importância que o especialista atribuiu àquela informação para o diagnóstico clínico de asma. 0 escore variou de 0 a 14 pontos. 0 estudo demonstrou que um escore $=5$ pontos permitiu discriminar pacientes asmáticos dos controles com sensibilidade de 93\% e especificidade de 100\%. Os autores concluíram que a validação de uma nota de corte permite uma interpretação alternativa às informações fornecidas pelo módulo de asma do ISAAC, levando em conta o conjunto das informações e não somente as respostas individuais de cada questão em estudos de prevalência de asma em adultos.

Este trabalho é um excelente exemplo de uma inicitiva na direção de se criar um instrumento confiável e validado para pesquisa da epidemiologia da asma em adultos no Brasil. 0 estudo é oportuno não apenas porque pontua o desconhecimento da prevalência de asma neste segmento da população brasileira como também porque oferece a opção de um instrumento de pesquisa conveniente, barato e de fácil utilização. Contudo, deve-se ter em mente alguns aspectos metodológicos que podem influenciar a interpretação dos resultados bem como a aplicabilidade do questionário em futuras pesquisas em estudos de campo.

Em primeiro lugar, trata-se de uma amostra de pacientes oriundos de um ambulatório de pneumologia dos quais 70\% eram portadores de asma moderada a grave e apenas 15\% eram portadores de asma leve. Embora os autores argumentem que é improvável que isto possa ter afetado o ponto de corte do escore, a interpretação dos resultados de futuros estudos epidemiológicos que utilizarem este escore deverá ser feita com cautela, pois é possível que casos de asma leve sejam subdiagnosticados. Uma abordagem alternativa foi aquela adotada por Grassi et al., ${ }^{(15)}$ que usaram um caminho inverso ao de Maçãira et al. para construir um escore a partir do ECRHS. No estudo relatado por Grassi, a construção do escore foi realizada a partir do exame de uma amostra aleatória de participantes do estudo epidemiológico (no qual o ECHRS foi aplicado) com e sem sintomas sugestivos de asma, os quais foram submetidos à investigação clínica (questionário estrurado) e funcional (espirometria e broncoprovocação com metacolina). 0 pon- to de corte do escore contruído por estes pesquisadores teve sensibilidade $(80,1 \%)$ e especificidade $(75,1 \%)$ inferiores às relatadas por Maiçãra et al., o que pode ser devido a diferenças nos questionários empregados (embora similares), na população selecionada para participar do estudo e na metodologia da construção do escore.

Em segundo lugar, os autores propõem uma modificação no questionário ISAAC acrescentando o termo "bronquite" à pergunta específica sobre o diagnóstico de asma, com o intuito de incluir aqueles pacientes portadores de asma rotulados como "bronquíticos". No entanto, a introdução desse termo como sinônimo de asma é questionável e, como os próprios autores chamam atenção, pode ter um efeito oposto ao esperado, levando ao aumento irreal da prevalência de asma. Por outro lado, o subdiagnóstico da asma tanto na infância quanto em adultos não é exclusivo do Brasil. A prevalência de asma diagnosticada é função de como se define o caso, o que, por sua vez, depende da morbidade da doença, da percepção do paciente ou de seus familiares, da percepção e concepção do médico assistente e do acesso do paciente ao sistema de saúde. Assim, não é difícil compreender porque a freqüência de sintomas de asma no estudo ISAAC Fase 1 foi superior à de asma diagnosticada, independentemente do local em que a pesquisa foi realizada. ${ }^{(5)}$ A análise do subgrupo de participantes com sibilos no último ano tem mostrado que, em crianças, o diagnóstico de asma é mais comum nos casos em que foram referidos quatro ou mais crises de sibilos por ano, sibilos provocando despertares noturnos e sibilos dificultando a fala. Ou seja, casos de asma mais leve (definida apenas por sintomas) naqueles estudos tiveram uma probabilidade maior de serem subdiagnosticados. ${ }^{(16)}$

Em conclusão, a determinação da prevalência de asma em adultos utilizando instrumentos acurados é necessária em nosso meio. 0 presente estudo representa uma primeira tentativa, ao validar uma forma distinta de interpretar as informações obtidas através do uso do questionário ISAAC. 0 próximo passo, como sugerido pelos autores, é a validação desse escore em estudos populacionais.

MÁRCIA MARGARET MENEZES PIZZICHINI NUPAIVA - Núcleo de Pesquisa em Asma e Inflamação das Vias Aéreas Deparmento de Clínica Médica. Centro de Ciências da Saúde da Universidade Federal de Santa Catarina - UFSC - Florianópolis (SC) Brasil 


\section{REFERÊNCIAS}

1. Sears MR. Epidemiology of childhood asthma. Lancet. 1997;350(9083):1015-20. Review.

2. Global Iniciative for Asthma - GINA. Global Strategy for asthma management and prevention [text on the Internet]. Bethesda (MD): National Institutes of Health; 2002 [cited 2003 Nov 20]. Available from <http:// www.ginasthma.com.

3. Sociedade Brasileira de Pneumologia e Tisiologia. 111 Consenso Brasileiro no Manejo da Asma. Capítulo 1. Definição, epidemiologia e patogenia. J Pneumol. 2002;28(1);S1-20.

4. Asher Ml, Keil U, Anderson HR, Beasley R, Crane J, Martinez $F$, et al. International Study of Asthma and Allergies in Childhood (ISAAC): rationale and methods. Eur Respir J. 1995;8(3):483-91.

5. Worldwide variations in the prevalence of asthma symptoms: the International Study of Asthma and Allergies in Childhood (ISAAC). ISAAC Steering Committee. Eur Respir J. 1998;12(2):315-35.

6. Worldwide variation in prevalence of symptoms of asthma, allergic rhinoconjunctivitis, and atopic eczema: ISAAC. The International Study of Asthma and Allergies in Childhood (ISAAC) Steering Committee. Lancet. 1998;351(9111):1225-32.

7. Solé D, Yamada E, Vana AT, Costa-Carvalho BT, Naspitz CK. Prevalence of asthma and related symptoms in school-age children in São Paulo, Brazil- International Study of Asthma and Allergies in Children (ISAAC). J Asthma. 1999;36(2):20512.

8. Ferrari FP, Rosário Filho NA, Ribas LFO, Callefe LG. Prevalência de asma em escolares de Curitiba - projeto ISAAC (International Study of Asthma and Allergies in Childhood). J Pediatr (Rio J). 1998;74(4):299-305.
9. Werneck G, Ruiz S, Hart R, White M, Romeu 1. Prevalence of asthma and other allergies in Brazilian schoolchildren. J Asthma. 1999;36(8):677-90.

10. de Britto MC, Bezzerra PG, Ferreira OS, Maranhao IC, Trigueiro GA. Asthma prevalence in schoolchildren in a city in north-east Brazil. Ann Trop Pediatr. 2000;20(2):95100.

11. Camargos PA, Castro RM, Feldman JS. [Prevalence of symptoms related to asthma in school children of Campos Gerais, Brazil]. Rev Panam Salud Publica. 1999;6(1):8-15. Spanish.

12. Piazza HE. Prevalência dos sintomas de asma e rinite em adolescentes da cidade de Florianópolis [tese]. Florianópolis: Universidade Federal de Santa CatarinaUFSC; 2001.

13. Burney PGL, Luczynska C, Chinn S, Jarvis D. The European Community Respiratory Health Survey. Eur Respir J. 1994;7(5):954-60.

14. Maçãira EF, Algranti E, Stelmach R, Ribeiro M, Nunes MPT, Mendonça EMC, et al. Determinação de escore e nota de corte do módulo de asma do International Study of Asthma and Allergies in Childhood para discriminação de adultos asmáticos em estudos epidemiológicos. J Bras Pneumol. 2005;31(6):47785.

15. Grassi M, Rezzani C, Biino G, Marinoni A. Asthma-like symptoms assessment through ECRHS screening questionnaire scoring. J Clin Epidemiol. 2003;56(3):23847.

16. Pizzichini MMM, Pizzichini E, Sears MR. Asthma diagnosis and severity of symptoms in Canadian children [abstract]. Eur Respir J. 1996;(Suppl 23):1216. 